09

\title{
Предельно короткие оптические импульсы в фотонном кристалле из углеродных нанотрубок под действием внешнего поля накачки
}

\author{
(C) И.С. Двужилов, Ю.В. Двужилова, М.Б. Белоненко \\ Волгоградский государственный университет, \\ 400062 Волгоград, Россия \\ e-mail: dvuzhilov.ilya@volsu.ru
}

Поступила в редакцию 27.01.2020 г.

В окончательной редакции 28.09.2020 г.

Принята к публикации 02.10.2020 г.

\begin{abstract}
Рассмотрена эволюция предельно коротких оптических импульсов в фотонном кристалле из полупроводниковых углеродных нанотрубок, помещенном во внешнее поле накачки. Показана возможность устойчивого распространения электромагнитных импульсов в условиях поля накачки и затухания за счет баланса диссипативных сил и внешнего поля. Продемонстрирована стабильность формы предельно короткого оптического импульса на больших временах при изменении различных параметров среды, таких как период неоднородности углеродных нанотрубок в фотонном кристалле и глубина модуляции показателя преломления.
\end{abstract}

Ключевые слова: фотонный кристалл, предельно короткие оптические импульсы, нелинейная среда, световые пули, углеродные нанотрубки.

DOI: $10.21883 /$ OS.2021.01.50445.14-20

\section{Введение}

Одной из основных задач современной нелинейной оптики является создание приборов для обработки, хранения и передачи информации, которые могут быть использованы в качестве элементной базы устройств нанофотоники, а также опто- и наноэлектроники. В основе таких оптических приборов могут лежать разнообразные эффекты, основанные на исследованиях взаимодействия электромагнитного излучения с веществом. Одними из таких веществ с заданными свойствами являются фотонные кристаллы, под которыми понимается среда с пространственно периодическим показателем преломления, в которой существует фотонная запрещенная зона. Таким образом, фотонный кристалл можно рассматривать как оптический фильтр, который способен пропускать фотоны с определенной частотой. $[1,2]$. Наличие неоднородности в фотонном кристалле обеспечивает идеальную нелинейную среду для распространения и исследования электромагнитных солитонов, предельно коротких оптических импульсов или световых пуль [3].

Также не утихает интерес к исследованию новых типов локализованных в пространстве фемтосекундных импульсов электрического поля длительностью, соответствующей нескольким периодам колебания поля, вся энергия которых остается сосредоточенной в конечной ограниченной области пространства [4,5]. Интерес к изучению распространения предельно коротких импульсов обусловлен, в частности, высокой направленностью их излучения, стабильностью формы и устойчивостью к возмущениям параметров, а также достижимыми на практике значениями пиковой интенсивности поля, при которых не происходит разрушения материала волновода, но уже в полной мере проявляются его нелинейные свойства [6-9].

Для устойчивого распространения необходим баланс между дисперсией и нелинейностью среды. Таким образом, углеродные нанотрубки [10-12], обладающие уникальными нелинейными свойствами вследствие непараболичности закона дисперсии для электронов (зависимость энергии от квазиимпульса), обусловливают нелинейность отклика нанотрубок на воздействие электромагнитных полей умеренных напряженностей, начиная со значений $10^{3}-10^{4} \mathrm{~V} / \mathrm{cm}$, и являются подходящей средой для распространения электромагнитных импульсов [13]. В то же время остаются без внимания задачи с диссипацией, вносимой углеродными нанотрубками, а также „подкачкой“ энергии, необходимой для ее компенсации. Благодаря учету диссипации и внешнего поля накачки, отпадет необходимость выполнения условия,

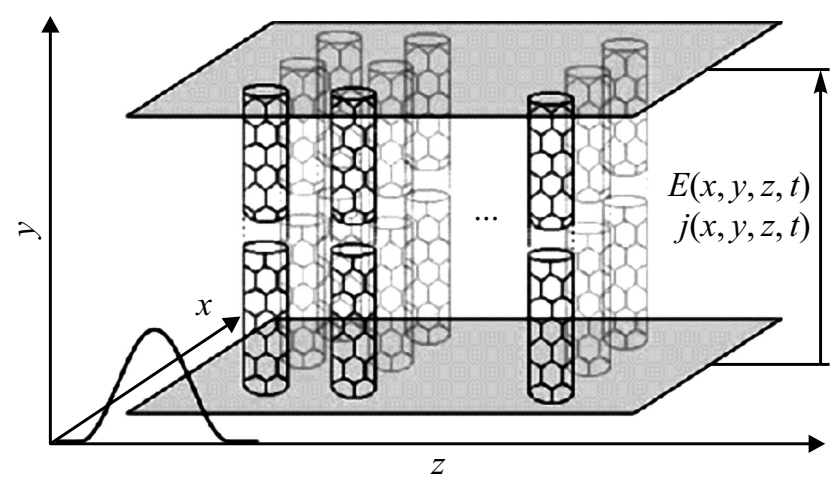

Рис. 1. Геометрия задачи. 

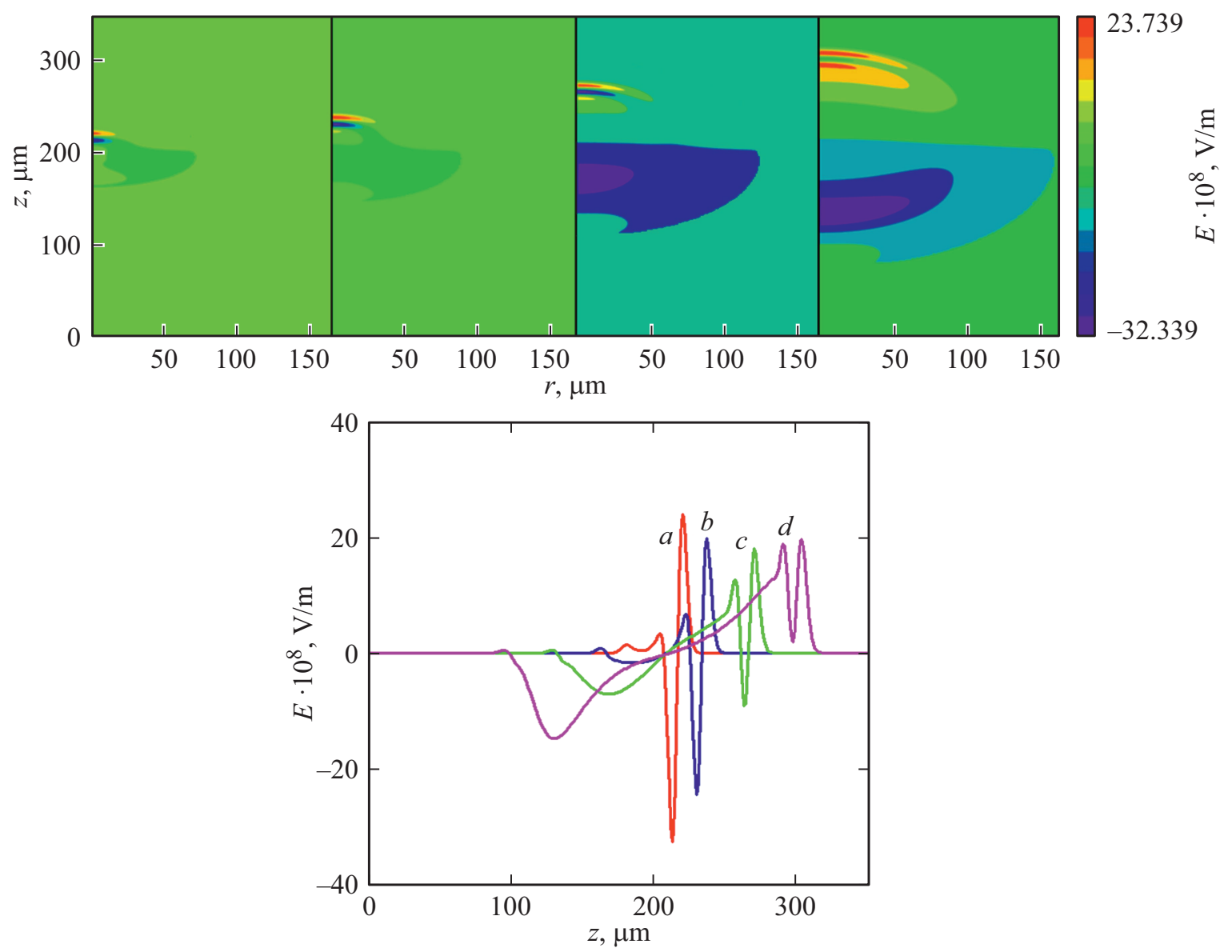

Рис. 2. Динамика трехмерного предельно короткого оптического импульса в различные моменты времени: $(a) 2,(b) 4,(c) 8$, (d) 12 ps. При следующих параметрах среды: глубина модуляции показателя преломления $\alpha=0.05$, период модуляции $\chi=2.5 \mu$ m. На верхнем рисунке - распределение поля в цилиндрической системе координат, на нижнем - сечения по оси $r=0$.

налагаемого на соотношение длительности импульса, времени релаксации в электронной подсистеме, а также интервала времени, в течение которого результаты моделирования могут считаться справедливыми. Выполнение данного критерия предполагало, что время релаксации существенно превышает длительность импульса, но при этом все еще меньше времени наблюдения за системой [14]. Таким образом, становится актуальным рассмотрение диссипативных эффектов, без которых не обходятся реальные устройства. Следовательно, задача с рассмотрением всех параметров, стабилизирующих и разрушающих предельно короткий импульс на больших временах, является весьма нетривиальной.

Следует отметить имеющиеся предпосылки для данной работы, а именно в работах [15-20] уже была установлена возможность устойчивого распространения двумерных и трехмерных предельно коротких оптических импульсов в средах ориентированных углеродных нанотрубок с пространственно переменным показателем преломления. Также были выявлены закономерности динамики данных импульсов в средах с меняющимся показателем преломления под действием внешних полей $[21,22]$ и показаны зависимости эволюции оптиче- ских импульсов от параметров среды (период и глубина модуляции показателя преломления).

Все вышеизложенные обстоятельства и послужили стимулом к проведению данного исследования.

\section{Основные уравнения}

Исследование электронной структуры углеродных нанотрубок (УНТ), как правило, проводится в приближении сильной связи в рамках анализа динамики $\pi$-электронов. Общее дисперсионное соотношение для структуры нанотрубок полупроводникового типа имеет следующий вид $[23,24]$ :

$$
\varepsilon_{s}(p)= \pm \gamma_{0} \sqrt{1+4 \cos (a p) \cos \left(\frac{\pi s}{m}\right)+4 \cos ^{2}\left(\frac{\pi s}{m}\right)},
$$

где $\quad \gamma_{0} \approx 2.7 \mathrm{eV}, \quad s=1,2 \ldots m, \quad a=3 b / 2 \hbar$, $b=0.142 \mathrm{~nm}-$ расстояние между соседними атомами углерода. Отметим, что $m$ имеет смысл числа гексагонов вдоль окружности нанотрубки.

Геометрия задачи (рис. 1) предполагает, что ток, приложенное электрическое поле и электрическое поле 

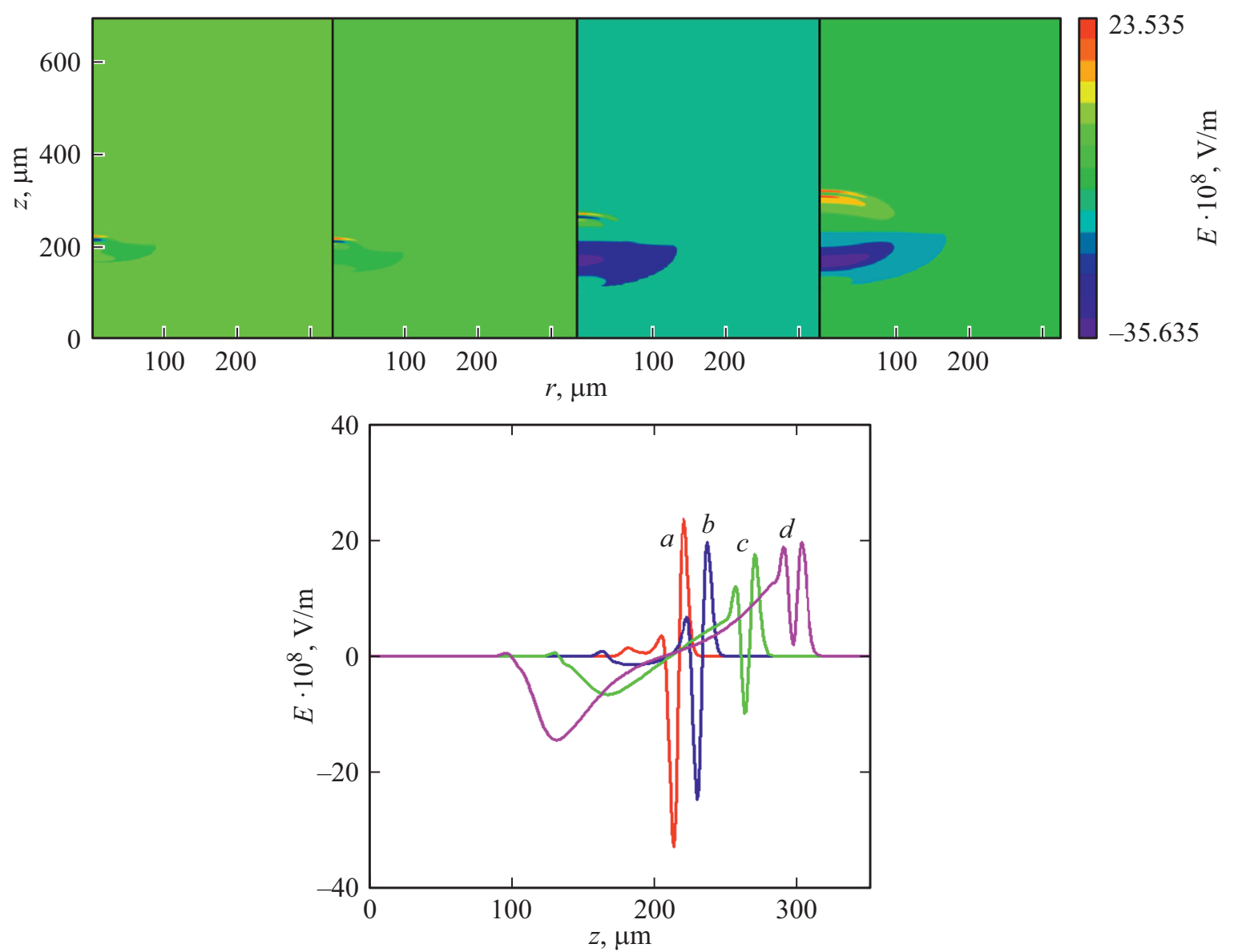

Рис. 3. Динамика трехмерного предельно короткого оптического импульса в различные моменты времени: (a) 2, (b) 4, (c) 8, (d) 12 ps. При следующих параметрах среды: глубина модуляции показателя преломления $\alpha=0.05$, период модуляции $\chi=5 \mu$ m. На верхнем рисунке - распределение поля в цилиндрической системе координат, на нижнем - сечения по оси $r=0$.

импульса направлены вдоль оси УНТ, а импульс движется перпендикулярно массиву нанотрубок. Отметим, что поскольку типичный размер УНТ и расстояние между ними много меньше, чем типичный размер пространственной области, в которой локализован предельно короткий импульс, можно использовать приближение сплошной среды и считать ток распределенным по объему. Вектор-потенциал имеет вид $\mathbf{A}=(0, A(x, y, z, t), 0)$, плотность электрического тока $\mathbf{j}=(0, j(x, y, z, t), 0)$. Поскольку типичное время релаксации для электронов в УНТ можно оценить как $3 \cdot 10^{-13} \mathrm{~s}$ [25], то ансамбль электронов на временах, типичных для задач динамики предельно короткого оптического импульса (порядка $10^{-14} \mathrm{~s}$ ), можно описывать при помощи бесстолкновительного кинетического уравнения Больцмана [26]:

$$
\frac{\partial f}{\partial t}-\frac{q}{c} \frac{\partial A_{s}}{\partial t} \frac{\partial f}{\partial p}=0,
$$

где $f=f\left(p_{s}, s, t\right)$ - функция распределения, неявно зависящая от координаты (в силу зависимости от координаты вектора-потенциала А), причем функция распределения $f$ в начальный момент времени совпадает с функцией $F_{0}-$ равновесной функцией распределения Ферми:

$$
F_{0}=\frac{1}{1+\exp \left\{E(\mathbf{p}) / k_{b} T\right\}},
$$

где $T$ - температура, $k_{b}$ - постоянная Больцмана.

Для плотности тока воспользуемся стандартным выражением [13]:

$$
j=2 e \sum_{s=1}^{m} \int_{Z B} v_{s}(p) f(p, s) d p,
$$

где введена групповая скорость электронов $v_{s}(p)=\frac{\partial \varepsilon_{s}(p)}{\partial p}, \varepsilon_{s}(p)-$ закон дисперсии, описывающий электронные свойства УНТ (1), e - заряд электрона. Интегрирование ведется по первой зоне Бриллюэна.

Групповую скорость с учетом закона дисперсии можно разложить в ряд Фурье:

$$
v_{z}(s, x) \sum_{m} a_{m s} \sin (m x),
$$

где

$$
a_{m s}=\frac{1}{\pi} \int_{-\pi}^{\pi} v_{z}(s, x) \sin (m x) d x
$$



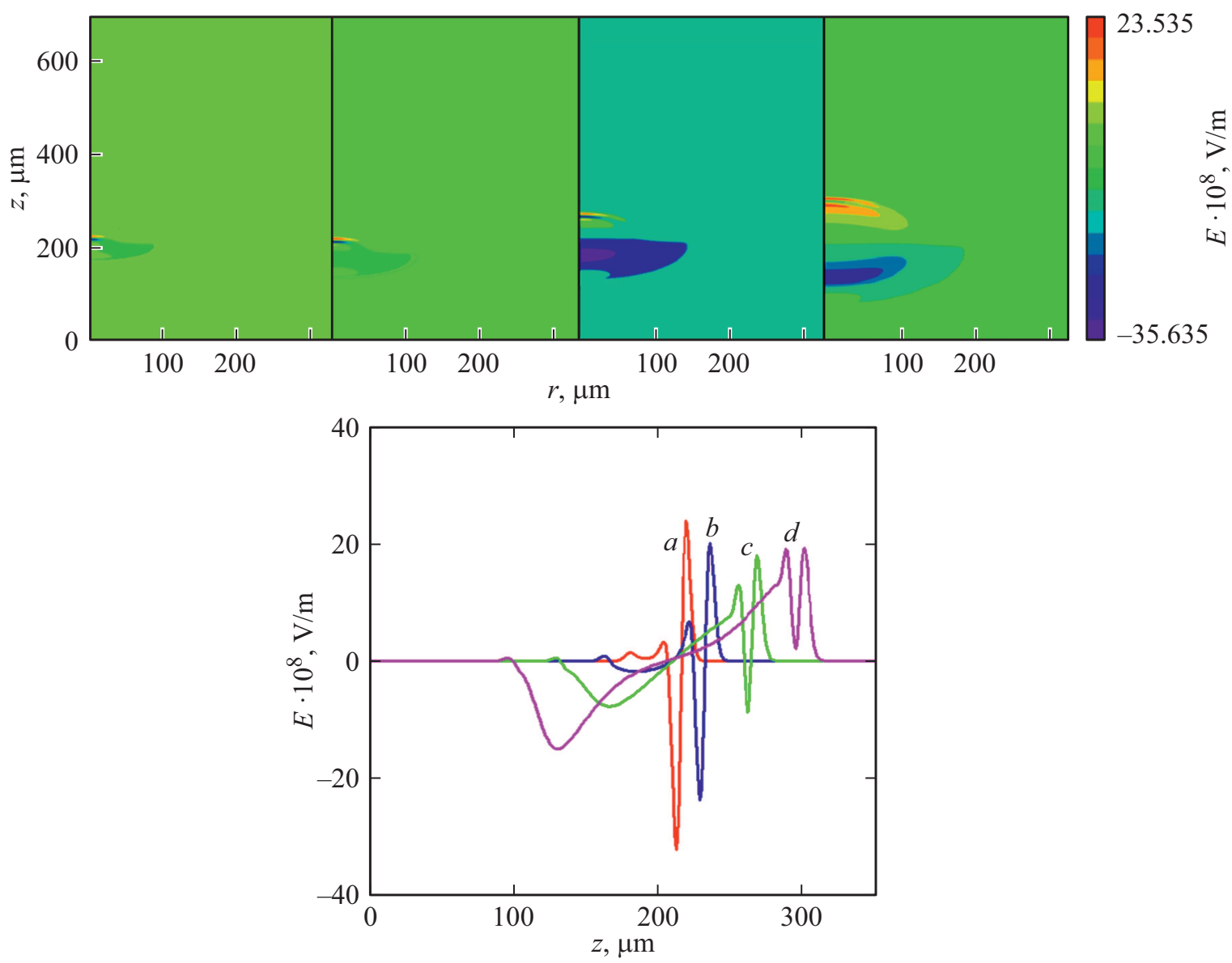

Рис. 4. Динамика оптического импульса в различные моменты времени: $(a) 2,(b) 4,(c) 8,(d) 12$ ps. При следующих параметрах среды: глубина модуляции показателя преломления $\alpha=0.05$, период модуляции $\chi=7.5 \mu \mathrm{m}$. На верхнем рисунке - распределение поля в цилиндрической системе координат, на нижнем - сечения по оси $r=0$.

- коэффициенты разложения, убывающие с ростом $m$. Здесь можно ограничиться первыми 15 неисчезающими слагаемыми [28] и получить широко применяемое в приложениях, но не интегрируемое методом обратной задачи рассеяния обобщенное уравнение sine-Gordon.

Уравнение на вектор-потенциал электромагнитного поля трехмерного предельно короткого оптического импульса, записанное в калибровке Кулона $(E=-\partial A / c \partial t)$, будет иметь вид

$$
\begin{gathered}
\frac{\partial^{2} \mathbf{A}}{\partial x^{2}}+\frac{\partial^{2} \mathbf{A}}{\partial y^{2}}+\frac{\partial^{2} \mathbf{A}}{\partial z^{2}}-\frac{n^{2}(x, y, z)}{c^{2}} \frac{\partial^{2} \mathbf{A}}{\partial t^{2}} \\
+\Gamma \frac{\partial \mathbf{A}}{\partial t}+\frac{4 \pi}{c} \mathbf{j}^{*} \phi(t)=0,
\end{gathered}
$$

где $n(x, y, z)$ - пространственно модулированный показатель преломления, $\Gamma-$ феноменологически введённый показатель накачки электрического поля (за счет различных механизмов). Отметим, что решение уравнения Больцмана со столкновительным членом в приближении времени релаксации приводит также к экспоненциальному уменьшению тока в зависимости от времени:

$$
\phi(t)= \begin{cases}0, & t<t_{0}(z), \\ \exp \left(-t / t_{\text {rel }}\right), & t \geq t_{0}(z)\end{cases}
$$

Здесь $t_{0}(z) \cong\left(z-z_{0}\right) / v-$ момент времени, в который интенсивность импульса на его переднем фронте, измеренная в точке с координатой $z$, в $e$ раз меньше пиковой интенсивности импульса, $z_{0}-$ начальная координата „центра масс“" импульса в начальный момент времени $t=0, v \cong c / \sqrt{k_{0}}-$ аппроксимация скорости импульса по порядку величины, $k_{0}-$ усредненная относительная диэлектрическая проницаемость среды (массива нанотрубок), $t_{\mathrm{rel}}$ - время релаксации электронной подсистемы УНТ. Отметим, что $\phi(t)$ представляет собой поправочный коэффициент к выражению для плотности тока в бесстолкновительном приближении, который записан эмпирически на основе обобщения и анализа результатов численного моделирования и представляет собой грубую аппроксимацию для плотности тока в приближении времени релаксации. Таким образом, мы хотели показать, что импульс тока, индуцируемый предельно коротким оптическим импульсом, затухает по 

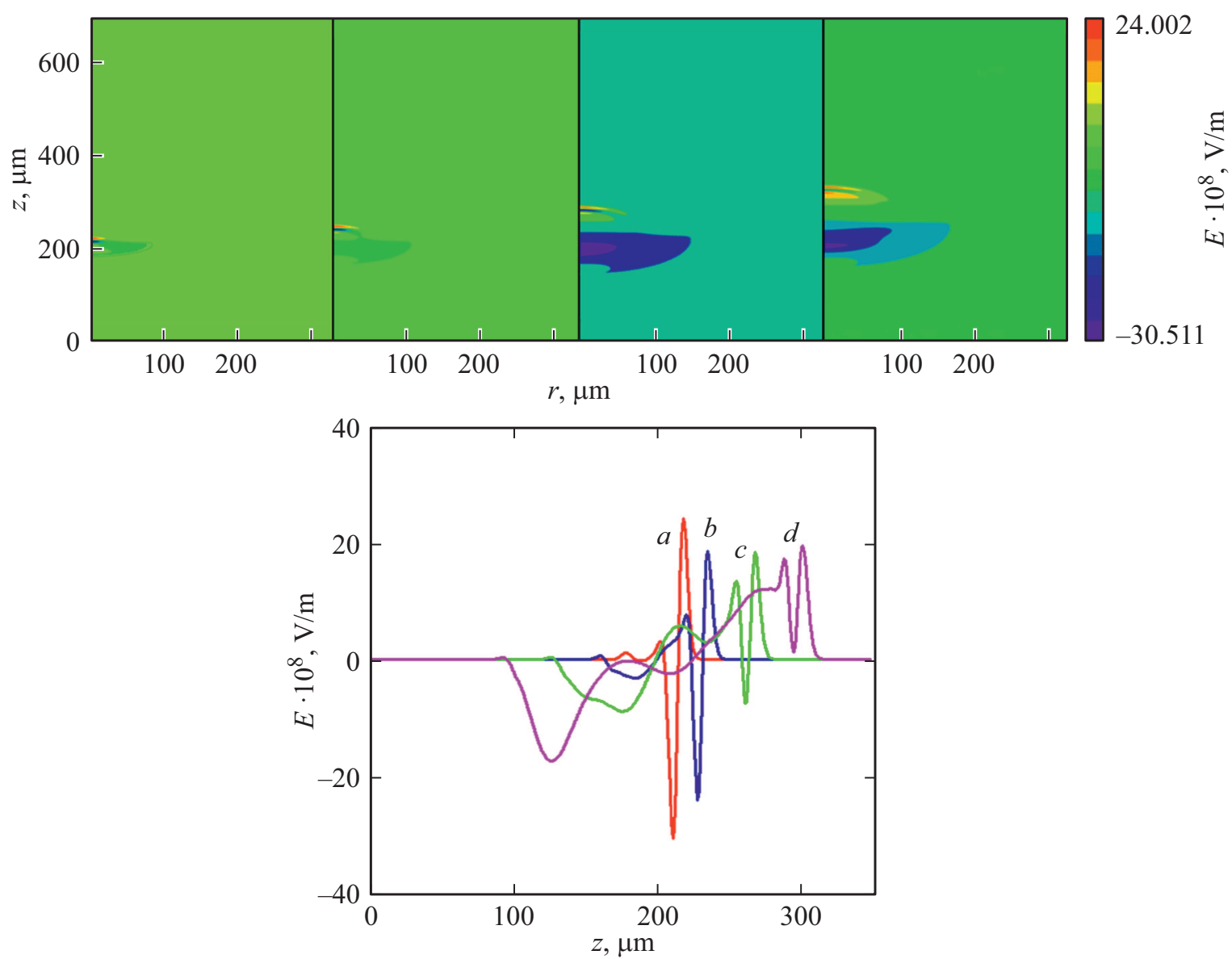

Рис. 5. Динамика трехмерного предельно короткого оптического импульса в различные моменты времени: (a) 2, (b) 4, (c) 8, (d) 12 ps. При следующих параметрах среды: глубина модуляции показателя преломления $\alpha=0.5$, период модуляции $\chi=2.5 \mu$ m. На верхнем рисунке - распределение поля в цилиндрической системе координат, на нижнем - сечения по оси $r=0$.

экспоненциальному закону при учете релаксационных процессов. Слагаемое, описывающее экспоненциальное затухание импульса, подробно описано в работе [29].

Для компенсации диссипативных эффектов и дифракционного расплывания импульса (т.е. импульс, вышедший (в перпендикулярном направлении к оси распространения) за пределы усиливающей области, затухнет) отвечает параметр Г накачки внешним электрическим полем, форма которого выбрана супергауссовой [30]:

$$
\Gamma(\tilde{r})=Q_{\Gamma} \exp \left(-\frac{\tilde{r}^{6}}{\tilde{l}_{\Gamma}}\right)
$$

Здесь параметр $\tilde{l}_{\Gamma}$ определяет ширину усиливающей среды в направлении, перпендикулярном направлению распространения импульса электрического поля, $Q_{\Gamma}$ - коэффициент усиления, вводимый феноменологически и зависящий от свойств усиливающей среды $\left(Q_{\Gamma}=0.0000001\right)$.

С учетом сказанного выше окончательное уравнение на вектор-потенциал в цилиндрической системе коорди- нат примет вид

$$
\begin{gathered}
\frac{\partial^{2} A_{z}}{\partial z^{2}}+\frac{1}{r} \frac{\partial}{\partial r}\left(r \frac{\partial A_{z}}{\partial r}\right)-\frac{n^{2}(z, r)}{c^{2}} \frac{\partial^{2} A_{z}}{\partial t^{2}}+\Gamma \frac{\partial \mathbf{A}}{\partial t} \\
+\frac{q}{\pi h} \phi(t) \sum_{m} c_{m} \sin \left(\frac{m a q}{c} A_{z}(t)\right)=0, \\
r=\sqrt{x^{2}+y^{2}} .
\end{gathered}
$$

Слагаемым, которое зависит от угла поворота, можно пренебречь [19].

Начальные условия на вектор-потенциал, соответствующие гауссову профилю импульса для одного колебания электрического поля, и показатель преломления среды заданы следующим образом:

$$
\begin{gathered}
A_{t=0}=A_{0} \exp \left\{-\frac{r^{2}}{\gamma^{2}}\right\} \exp \left\{-\left(z-z_{c}\right)^{2} / \beta^{2}\right\} \\
\left.\frac{d A}{d t}\right|_{t=0}=\frac{2 v z}{\gamma^{2}} A_{0} \exp \left\{-\frac{r^{2}}{\gamma^{2}}\right\} \exp \left\{-\left(z-z_{c}\right)^{2} / \beta^{2}\right\} \\
n(z, r)=1+a \cos (2 \pi z / \chi) .
\end{gathered}
$$



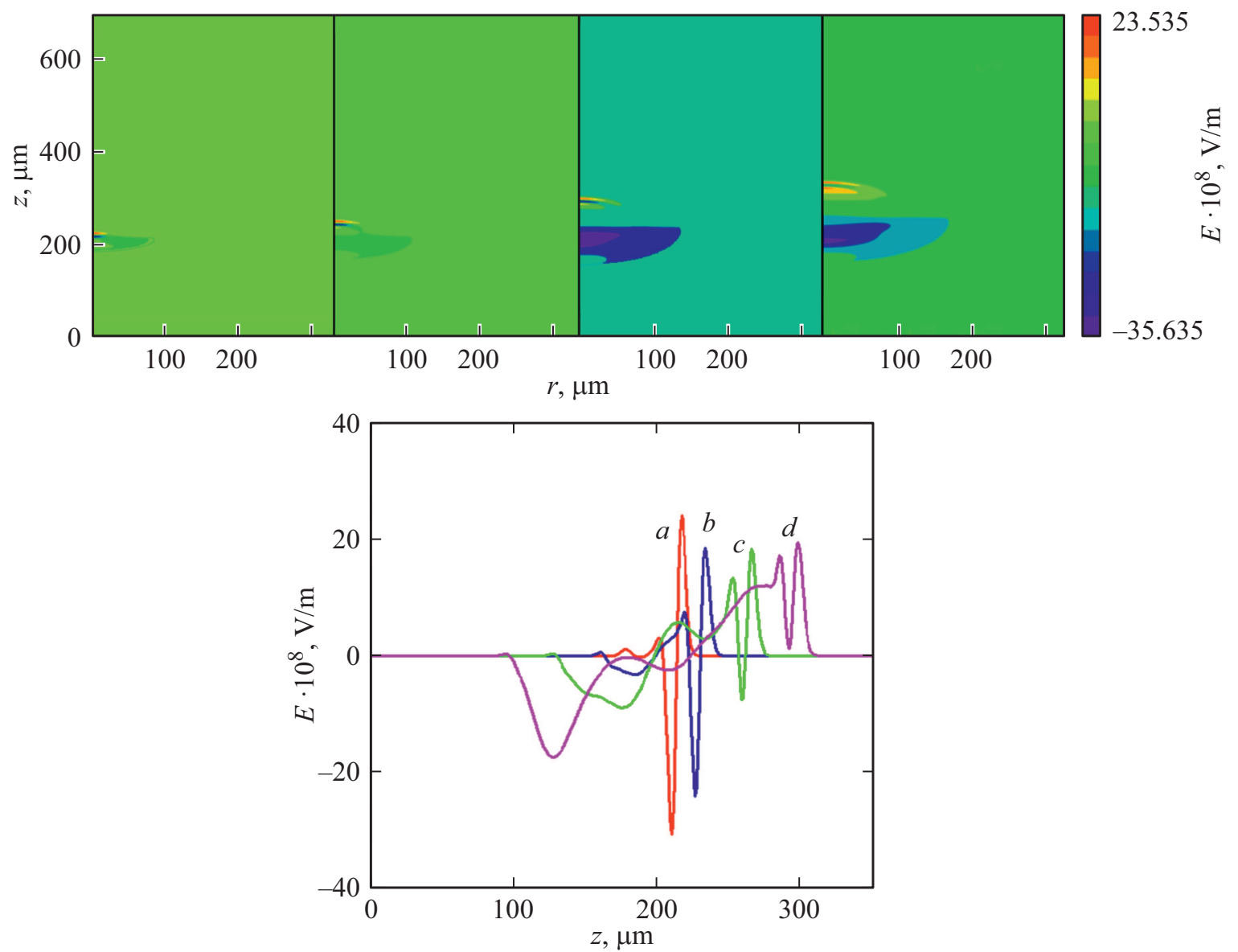

Рис. 6. Динамика трехмерного предельно короткого оптического импульса в различные моменты времени: $(a) 2,(b) 4,(c) 8$, (d) 12 ps. При следующих параметрах среды: глубина модуляции показателя преломления $\alpha=0.25$, период модуляции $\chi=2.5 \mu$ m. На верхнем рисунке - распределение поля в цилиндрической системе координат, на нижнем - сечения по оси $r=0$.

Здесь $\beta, \gamma$ - параметры, определяющие ширину импульса по осям $z$ и $r$ соответственно, $t_{0}$ - начальный момент времени, $v-$ начальная скорость импульса при входе в среду, $\alpha-$ глубина модуляции показателя преломления, $\chi-$ период модуляции показателя преломления. Значения параметров, определяющих ширину импульса, а также начальную скорость импульса при входе в среду, задавались следующим образом:

$$
\beta=\gamma=\sqrt{1-v^{2}}, \quad v=0.95 c .
$$

\section{Численное моделирование и обсуждение результатов}

Исследуемое уравнение (8) было решено численно с помощью явной разностной схемы типа „крест“ [31]. В работах $[15,19]$ показаны результаты динамики двумерного и трехмерного предельно коротких оптических импульсов в среде ориентированных углеродных нанотрубок, которая имеет пространственно модулированный показатель преломления. Здесь (рис. 2-7) представлены результаты эволюции импульса электромагнитного поля при его распространении в фотонном кристалле из УНТ в условиях внешнего электрического поля накачки в случае одного колебания электрического поля. На рис. 2-4 показаны результаты для одинаковой глубины модуляции показателя преломления $(\alpha) 0.05$ и различных периодов модуляции показателя преломления фотонного кристалла $(\chi): 2.5,5,7.5 \mu$ m соответственно.

На рис. 5-7 показаны результаты эволюции трехмерных предельно коротких импульсов в среде УНТ с внешним полем накачки и диссипацией. Здесь один и тот же период модуляции показателя преломления $(\chi) 2.5 \mu \mathrm{m}$ и различные значения глубины модуляции показателя преломления $(\alpha): 0.5,0.25,0.75$ соответственно.

Из рис. 2-7 можно сделать вывод о том, что энергия импульса остается локализованной в ограниченной пространственной области, импульс распространяется устойчиво. Однако вследствие дифракционных эффектов имеет место затухание, а на заднем фронте возникает „хвост“. Из рисунков видно, что форма импульса в целом сохраняется, однако присутствует незначительное искривление на фронте импульса, что в свою очередь связано с дифракцией.

В ходе работы также было выявлено, что незначительное изменение параметра накачки (параметр накачки 

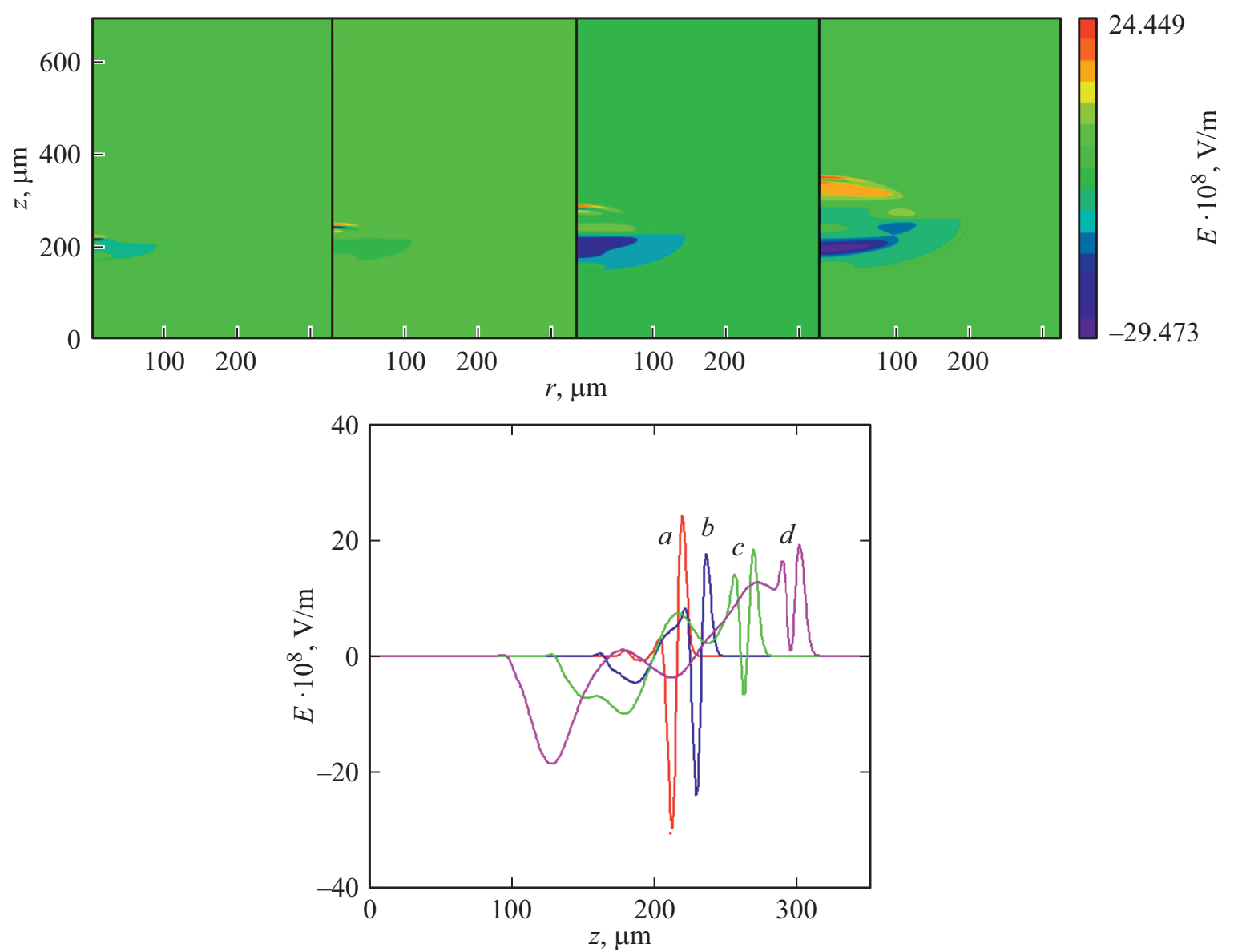

Рис. 7. Динамика трехмерного предельно короткого оптического импульса в различные моменты времени: (a) 2, (b) 4, (c) 8, (d) 12 ps. При следующих параметрах среды: глубина модуляции показателя преломления $\alpha=0.75$, период модуляции $\chi=2.5 \mu$ m. На верхнем рисунке - распределение поля в цилиндрической системе координат, на нижнем - сечения по оси $r=0$.

менялся в пределах 10\%) в целом не изменяет формы, амплитуды и групповой скорости импульса, что в свою очередь еще раз подтверждает устойчивость его распространения.

Из проведенных исследований можно сделать следующий вывод: имеет место возможность устойчивого распространения предельно короткого оптического импульса (электрическое поле остается локализованным в ограниченной пространственной области), обусловленная балансом двух процессов - накачки и затухания, даже в случае небольшого возмущения параметра накачки. Дисперсионное расплывание импульсов в направлении, перпендикулярном оси распространения, в ходе его динамики можно компенсировать вводом накачки, неоднородной вдоль диаметра образца. Импульсы в этом случае перестают уширяться. Дисперсионное расплывание вдоль оси образца компенсируется нелинейностью среды углеродных нанотрубок за счет непараболичности закона дисперсии электронов, находящихся в зоне проводимости. Отметим также, что „закон сохранения площади импульса“ [32] в данном случае не выполняется вследствие наличия усиления.

\section{Финансирование работы}

Авторы выражают благодарность Министерству науки и высшего образования РФ за поддержку в рамках государственного задания численного моделирования (проект № 0633-2020-0003).

\section{Конфликт интересов}

Авторы заявляют, что у них нет конфликта интересов.

\section{Список литературы}

[1] Mekis A., Chen J.C., Kurland I., Fan S., Villeneuve P.R., Joannopoulos J.D. // Phys. Rev. Lett. 1996. V. 77. P. 3787. doi 10.1103/PhysRevLett.77.3787

[2] Tokushima M., Kosaka H., Tomita A., Yamada H. // Appl. Phys. Lett. 2000. V. 76. P. 952.

[3] Sazonov S.V., Ustinov N.V. // Phys. Rev. A. 2018. V. 98. P. 063803-1-12. doi 10.1103/PhysRevA.98.063803

[4] Fibich G., Ilan B. // Opt. Lett. 2004. V. 29. P. 887-889. doi https://doi.org/10.1364/OL.29.000887

[5] Goorjian P.M., Silberberg Y. // J. Opt. Soc. Am. B. 1997. V. 14. P. 3253-3260. doi https://doi.org/10.1364/JOSAB.14.003253 
[6] Желтиков А.М. // УФН. 2007. Т. 177. С. 737; Zheltikov A.M. // Phys. Usp. 2007. V. 50. P. 705. doi https://doi.org/10.3367/UFNr.0177.200707d.0737

[7] Mihalache D. // Rom. J. Phys. 2017. V. 69. P. 403.

[8] Mourou G., Mironov S., Khazanov E., Sergeev A. // Eur. Phys. J. Spec. Top. 2014. V. 223. P. 1181. doi 10.1140/epjst/e2014-02171-5

[9] Pakhomov A.V., Arkhipov R.M., Babushkin I.V., Arkhipov M.V., Tolmachev Yu.A., Rosanov N.N. // Phys. Rev. A. 2017. V. 95. P. 013804. doi 10.1103/PhysRevA.95.013804

[10] Рокко М.К., Уильямс Р.С., Аливисатос П. Нанотехнология в ближайшем десятилетии. Прогноз направления исследований. М.: Мир, 2002. 292 с.

[11] Харрис П. Углеродные нанотрубы и родственные структуры. Новые материалы XX века. М.: Техносфера, 2003. $336 \mathrm{c}$.

[12] Maksimenko S.A., Slepyan G.Ya. Handbook of nanotechnology. Nanometer structure: theory, modeling and simulation. Bellingham: SPIE Press, 2004. 145 p.

[13] Белоненко М.Б., Демушкина Е.В., Лебедев Н.Г. // ФТТ. 2008. T. 50. C. 368.; Belonenko M. et al. // J. Rus. Las. Res. 2006. V. 27. P. 457.

[14] Zhukov A.V., Bouffanais R., Fedorov E.G., Belonenko M.B. // J. Appl. Phys. 2013. V. 114. P. 143106. doi 10.1063/1.4824370

[15] Zhukov A.V., Bouffanais R., Belonenko M.B., Konobeeva N.N., Nevzorova Y.V., George T.F. // Eur. Phys. J. D. 2015. V. 69. P. 129. doi 10.1140/epjd/e2015-50895-y

[16] Невзорова Ю.В., Белоненко М.Б., Галкина Е.В. // Изв. РАН Сер. физ. 2016. Т. 80. № 7. С. 916.; Nevzorova J.V., Belonenko M.B., Galkina E.N. // Izvestiya RAN. Seriya Fizicheskaya. 2016. V. 80. P. 837. doi 10.3103/S1062873816070182

[17] Belonenko M.B., Dvuzhilov I.S., Tuzalina O.Yu. // J. Nano-Electron. Phys. 2015. V. 7. N 4. P. 04013.

[18] Belonenko M.B., Dvuzhilov I.S., Galkina E.N., Nevzorova Y.V. // Mod. Phys. Lett. B. 2016. V. 30. P. 1650345. doi 10.1142/S0217984916503450

[19] Zhukov A.V., Bouffanais R., Belonenko M.B., Dvuzhilov I.S., Nevzorova Y.V. // Appl. Phys. B. 2017. V. 123. P. 196. doi 10.1007/s00340-017-6767-y

[20] Nevzorova Yu.V., Dvuzhilov I.S., Boroznin S.V., Zaporotskova I.V., Belonenko M.B. // EPJ Web of Conf. 2017. V. 161. P. 02018. doi 10.1051/epjconf/201716102018

[21] Dvuzhilova Y.V., Dvuzhilov I.S., Ten A.V., Boroznina E.V., Belonenko M.B. // Mod. Phys. Lett. B. 2019. V. 33. P. 1950275. doi 10.1142/S0217984919502750

[22] Елецкий А.В. // УФН. 1997. Т. 167. С. 945; Eletskii A.V. // Physics - Uspekhi. 1997. V. 40. P. 899.

[23] Dresselhaus M.S., Dresselhaus G., Eklund P.C. Science of Fullerenes and Carbon Nanotubes. Academic Press, 1996. $965 \mathrm{p}$.

[24] Tans S.J., Devoret M.H., Dai H. et al. // Nature. 1997. V. 386. P. 474.

[25] Ландау Л.Д., Лифшии Е.М. Физическая кинетика. М.: Физматлит, 1979. $275 \mathrm{c}$.

[26] Zhukov A.V., Bouffanais R., Fedorov E.G., Belonenko M.B. // J. Appl. Phys. 2013. V. 114. P. 143106.

[27] Буллаб Р., Кодри Ф. Солитоны. М.: Мир, 1983. 408 с.

[28] Ландау Л.Д., Либшии, Е.M. Теория упругости. М.: Наука, 1987. $248 \mathrm{c}$.
[29] Konobeeva N.N., Fedorov E.G., Rosanov N.N., Zhukov A.V., Bouffanais R., Belonenko M.B. // J. Appl. Phys. 2019. V. 126. P. 203103. doi.org/10.1063/1.5128365

[30] Svelto O. Principles of Lasers. NY.: Plenum, 1976. P. 620; Звелто О. Принципы лазеров. СПб.: Лань, 2008. С. 720.

[31] Бахвалов Н.С. Численные методы (анализ, алгебра, обыкновенные дифференциальные уравнения). М.: Наука, 1975. $632 \mathrm{c}$.

[32] Розанов Н.Н., Архипов Р.М., Архипов М.В. // УФН. 2018. T. 188. № 12. C. 1347. 